\title{
A smooth chaotic map with parameterized shape and symmetry
}

\author{
Daniel P. B. Chaves, Carlos E. C. Souza ${ }^{*}$ and Cecilio Pimentel
}

\begin{abstract}
We introduce in this paper a new chaotic map with dynamical properties controlled by two free parameters. The map definition is based on the hyperbolic tangent function, so it is called the tanh map. We demonstrate that the Lyapunov exponent of the tanh map is robust, remaining practically unaltered with the variation of its parameters. As the main application, we consider a chaotic communication system based on symbolic dynamics with advantages over current approaches that use piecewise linear maps. In this context, we propose a new measure, namely, the spread rate, to study the local structure of the chaotic dynamics of a one-dimensional chaotic map.
\end{abstract}

Keywords: Chaotic maps, Chaos-based modulation, Discrete-time chaotic systems, Lyapunov exponent, Orbit diagram, Nonlinear systems

\section{Introduction}

Chaotic signals are characterized by irregularity, aperiodicity, decorrelation, and broadband. They can be generated through simple deterministic dynamical systems [1] and have promising applications in cryptography [2], random number generation $[3,4]$, watermarking [5], communication [6,7], and systems modeling [8].

The well-known properties of decorrelation and broadband of chaotic signals make them good candidates to be used in direct sequence spread spectrum systems $[1,9]$. So, the chaos-based communication systems offer advantages as resistance to jamming, robustness in multi-path environments [7, 10], low probability of interception [11]. In addition, the complex-like behavior of chaotic signals propitiates secrecy in a physical level, and the easy-to-generate property of these signals offers a potential solution to implement low-cost systems [2, 12, 13]. Several coherent and non-coherent chaotic modulation schemes have been proposed in the literature. In the case of coherent modulation, the system requires a perfect knowledge of the chaotic sequence to demodulate the received signal. The detection is performed by using correlator-type receivers and this implies in a more complex communication system. Some well-known coherent

*Correspondence: carlos.ecsouza@ufpe.br

Federal University of Pernambuco, 1235 Prof. Moraes Rego Ave, 50670-901

Recife, Pernambuco, Brazil modulations schemes are chaos shift keying (CSK) [11] and chaos-based DS-CDMA [14], where chaotic sequences are used to spread data signals. On the other hand, noncoherent modulation mitigates synchronization problems of chaotic oscillators at the receiver. This less complex nature leads to intense study of this modulation scheme such as differential chaos shift keying (DCSK) [11]. In DCSK systems, each transmitted symbol is represented by two consecutive chaotic samples. The first one is the reference sample and the second one, which carries the data, is exactly the reference sample or its inverse, according to the information symbol to be transmitted. Other non-coherent modulation schemes have been proposed to overcome the decrease of the data rate and to improve the system performance. In the quadratic chaos shift keying (QCSK) [15], the system modulate digital information using orthogonal chaotic basis functions. In high efficiency DCSK (HE-DCSK) [16], the receiver recycles each reference sample to carry two bits of data when one data sample is modulated. The bandwidth efficiency is increased and the system becomes more robust to interception. Multi-carrier DCSK (MC-DCSK) [17] is a generalization of the DCSK to multiuser systems. An alternative solution, called OFDM-DCSK, was proposed in [18]. In the improved DSCK (I-DCSK) [19], the reference signal is added to the data carrier signal after time reversal in order to decrease the duration of the transmitted symbol by half of time and improve the spectral efficiency. Another 
recently proposed scheme is short reference DCSK (SRDCSK) [20], which uses reference samples of reduced size when compared to DCSK to increase data rate and enhance energy efficiency of the system.

In this paper, we focus on chaotic maps with suitable properties for chaotic modulation schemes based on symbolic dynamics [21]. There are two main schemes for chaos-based communication. In the first one, the information symbols are encoded into a chaotic sequence by a forward iteration of a chaotic map [22, 23], while in the second one, the information encoding uses backward iteration together with the symbolic dynamics of the orbits [21]. Two characteristics are worth mentioning about the latter approach. Firstly, it is capable of avoiding problems with error amplification and precision truncation found in the former scheme, and if implemented with adequate maps, it permits to trade performance and security. In this context, a chaotic communication system based on a piecewise linear chaotic map with a parameter that controls the length of a guard region (never visited region) was proposed in [24]. The same map has been considered in other chaos-based communication scenarios [25-27]. The guard region restricts the chaotic system dynamics, since the allowed orbits should never visit it. This limitation is eliminated by the new chaotic map proposed in this work.

The contribution of this paper is fourfold. First, we propose and analyze a one-dimensional chaotic map based on the tangent hyperbolic function (called tanh map) with suitable properties for chaotic modulation schemes based on symbolic dynamics [21].

The tanh map is specified by two parameters that permit to control the symmetry of the map (even or odd) and its shape. The latter has strong implication on the invariant distribution of the tanh map and may be used to generate a seldom visited region that works as a guard region on chaos-based communication schemes. Second, we study the Lyapunov exponent of the tanh map in terms of the control parameters and show that, in spite of the great impact of these parameters on the dynamics, the Lyapunov exponent is slightly affected by them. Third, we also analyze the bit error rate of a chaotic-based communication system using the tanh map over an additive white Gaussian channel and show that, with an adequate set of parameters, it outperforms the widely used chaotic map based on a piecewise linear function. Finally, we propose a new measure inspired by the fractal structure of the attractor. This captures the concentration of the system outputs over the attractor and is called spread rate.

The rest of this paper is organized in six sections. Section 2 contains background material on chaotic systems and chaotic modulation based on symbolic dynamics. Section 3 introduces the tanh map. The bit error rate analysis is conducted in Section 4. The Lyapunov exponent of this map is studied in Section 5. Section 6 proposes a new measure for analyzing the chaotic behavior of the proposed map, and Section 7 summarizes the conclusions of this work.

\section{Symbolic dynamics-based chaotic communication system \\ 2.1 Chaotic maps}

Unidimensional chaotic maps generate a discrete-time series $\left\{x_{i}\right\}_{i=0}^{\infty}$ obtained by the direct forward iteration of a suitable nonlinear and noninvertible function $f(x)$, such that

$$
x_{n}=f\left(x_{n-1}\right), \quad n=1,2, \ldots
$$

Alternatively, the time series is obtained by the recursive application of $f(x)$ over the initial condition $x_{0}$, which produces the orbit of $x_{0}$ under $f(x)$, that is, $\left\{x_{0}, f\left(x_{0}\right), f^{2}\left(x_{0}\right), \ldots\right\}$, where $f^{k}(x)$ denotes the $k$ th functional composition of $f(x)$.

\subsection{Chaotic modulation based on symbolic dynamics}

The application of symbolic dynamics in digital chaotic communications began with the works in [22, 28], where the information is transmitted by a slight perturbation of the orbit of an autonomous chaotic system. In order to introduce this method, we consider the modified Bernoulli map [24]

$$
f(x)=\left\{\begin{array}{lll}
\frac{2 x+(1+p)}{1-p}, & -1 \leq x \leq-p & \left(I_{0}\right) \\
\frac{x}{p}, & -p<x<p & \left(I_{1}\right) \\
\frac{2 x-(1+p)}{1-p}, & p \leq x \leq 1 & \left(I_{2}\right) .
\end{array}\right.
$$

Observe that $f(x)$ is defined over a bounded interval $\mathbb{H}$ and that $f(\mathbb{H})=\mathbb{H}$, which means that $\mathbb{H}$ is invariant under $f$. This map is piecewise linear and determines a partition of $\mathbb{H}$ into the subintervals $I_{0}, I_{1}$, and $I_{2}$. The chaotic modulation based on direct forward iteration of the expanding map given in (2) presents two drawbacks: error amplification and precision truncation [24].

These weakness can be overcome by generating the samples of the chaotic sequence by backward iteration [21]. Now, the map in (2) is exchanged by the inverse of the modified Bernoulli map

$$
f_{s}^{-1}(x)=\left\{\begin{array}{lll}
\frac{(1-p) x-(1+p)}{2}, & s=0 & \left(I_{0}\right) \\
p x, & s=1 & \left(I_{1}\right) \\
\frac{(1-p) x+(1+p)}{2}, & s=2 & \left(I_{2}\right)
\end{array}\right.
$$

that is a set of contraction maps over $\mathbb{H}$ indexed by the symbol $s$. The chaotic modulation of a finite sequence of symbols $s_{n}, s_{n+1}, \ldots, s_{M-1}$ occurs by iterating (3) from a known final condition $x_{N}$. 
The use of a contraction map mitigates the problems of the forward iteration associated to the numerical instability and alleviates the issues of synchronization typically found in chaotic communications [21, 23, 24].

The map (3) can be used to modulate information sequences [24-26]. The idea is to map the information bit 0 to $s=0$ and the information bit 1 to $s=2$, then the inner region $I_{1}$ for $s=1$ is never visited and is used as a guard region that ensures a minimum distance between transmitted sequences. Observe that the length of the guard region is increased with the increase of $p$, making the system more robust against noise. However, the system becomes more predictable, which decreases its security for cryptography. Another drawback of this map is the weakening of its chaotic properties, since the degree of freedom of the system is reduced by forbidding the occurrence of values in $I_{1}$.

In the next section, we present a new chaotic map that overcomes these drawbacks by maintaining a region that is seldom visited without damaging the chaotic properties of the original forward map.

\section{The tanh map}

The proposed chaotic map, the tanh map, is based on the hyperbolic tangent function and may have even or odd symmetry. The analytical description of the map $f$ : $[-1,1] \rightarrow[-1,1]$ is defined as

$$
f(x)= \begin{cases}e \cdot \tanh (r \cdot(x+1))-1, & x<0\left(I_{0}\right) \\ (-1)^{b} \cdot(e \cdot \tanh (-r \cdot(x-1))-1), & x \geq 0\left(I_{1}\right)\end{cases}
$$

where $e=2 / \tanh (r)$ is a scaling factor dependent on $r$, for $r>0$. Consequently, the tanh map depends on two control parameters, $b$ (determines the map symmetry) and $r$ (controls the shape of the map). When $b=0$ (resp. $b=1$ ), the tanh map has even (resp. odd) symmetry around $x=0$ and is denoted by e-tanh (resp. o-tanh). Applying the firstorder Taylor series approximation $\tanh (y) \cong y$ to (4) for sufficiently small values of its argument, the following map is derived for small values of $r$

$$
f(x)=\left\{\begin{array}{lll}
2 x+1, & x<0 & \left(I_{0}\right) \\
(-1)^{b} \cdot(-2 x+1), & x \geq 0\left(I_{1}\right)
\end{array}\right.
$$

which is the tent map for $b=0$ and the Bernoulli map for $b=1$. Figure 1 shows the e-tanh map for three values of $r, r=0.1,3,8$ as well as a finite sample of an orbit for each value of $r$. Figure 2 illustrates the o-tanh map and its orbits for the same values of $r$.

The inverse of the tanh map is

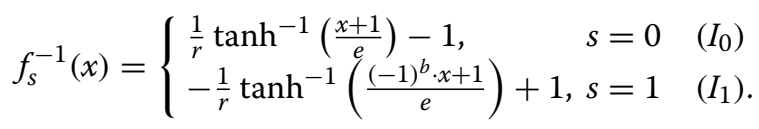

In the modulation process through a backward iteration, the information bit 0 is mapped to $s=0$ and the information bit 1 is mapped to $s=1$.

In Figs. 3 and 4, the asymptotic invariant distribution, called $\mu(x)$, for the e-tanh and o-tanh maps, respectively, is plotted for two values of $r$. A clear asymmetry of this distribution around $x=0$ for the e-tanh map is observed, while this is symmetric around $x=0$ for the o-tanh map. A common feature of the tanh map is the steady increase of points generated around its extreme values, which results in a seldom occurrence of values around $x=0$. Points $x_{k}$ in this region are generated with low probability and can then be interpreted as a guard region. The parameter $r$ controls the extension of this region.

The hyperbolic tangent function can be electronically obtained as the collector current of an emitter-coupled pair [29], and from it, the tanh map can be implemented by using the current-mode approach. More specifically, the collector current $I_{\mathrm{c} 2}$ of a differential pair with BJT may be expressed as a hyperbolic tangent function of its differential input voltage $v_{\text {id }}$ [29]

$$
I_{\mathrm{C} 2}=\frac{I_{\mathrm{EE}}}{2} \cdot\left[1-\tanh \left(\frac{v_{\mathrm{id}}}{2 V_{\mathrm{T}}}\right)\right]
$$

where $V_{\mathrm{T}}$ is the thermal voltage and $I_{\mathrm{EE}}$ is the polarization current of the differential pair. The function $f(x)$ is associated with the output current $I_{\text {out }}$ determined from the relation $I_{\text {out }}+I_{c 2}=I^{\prime}$, where $I^{\prime}$ is a constant that depends on the value of $r$. Thus, the output current is given by

$$
I_{\mathrm{out}}=\frac{I_{\mathrm{EE}}}{2} \cdot \tanh \left(\frac{v_{\mathrm{id}}}{2 V_{\mathrm{T}}}\right)-\beta
$$

where $\beta=I^{\prime}-I_{\mathrm{EE}} / 2$. Thus, the parameter $r$ can be obtained from $I_{\mathrm{EE}}$ and $\beta$.

\section{Bit error rate analysis}

The chaotic communication system proposed in [24] is composed of a symbolic dynamics chaotic modulator based on the backward iteration of the modified Bernoulli map, a communication channel, and a decoder that implements the Viterbi algorithm over a two-state trellis to estimate the transmitted sequence. This chaotic communication system is also considered in other communication scenarios [25-27]. These works analyze the impact of the length of the guard region given by the parameter $p$ on the system performance using computer simulations. In this subsection, we perform a similar analysis for the tanh map over the AWGN channel, as illustrated in Fig. 5.

Let $\left[s_{0}, s_{1}, \cdots, s_{N-1}\right]$ be a binary information sequence, $s_{k} \in\{0,1\}$. The chaotic sequence $\left[x_{0}, x_{1}, \cdots, x_{N}\right]$ is generated from the backward iteration of the tanh map starting 

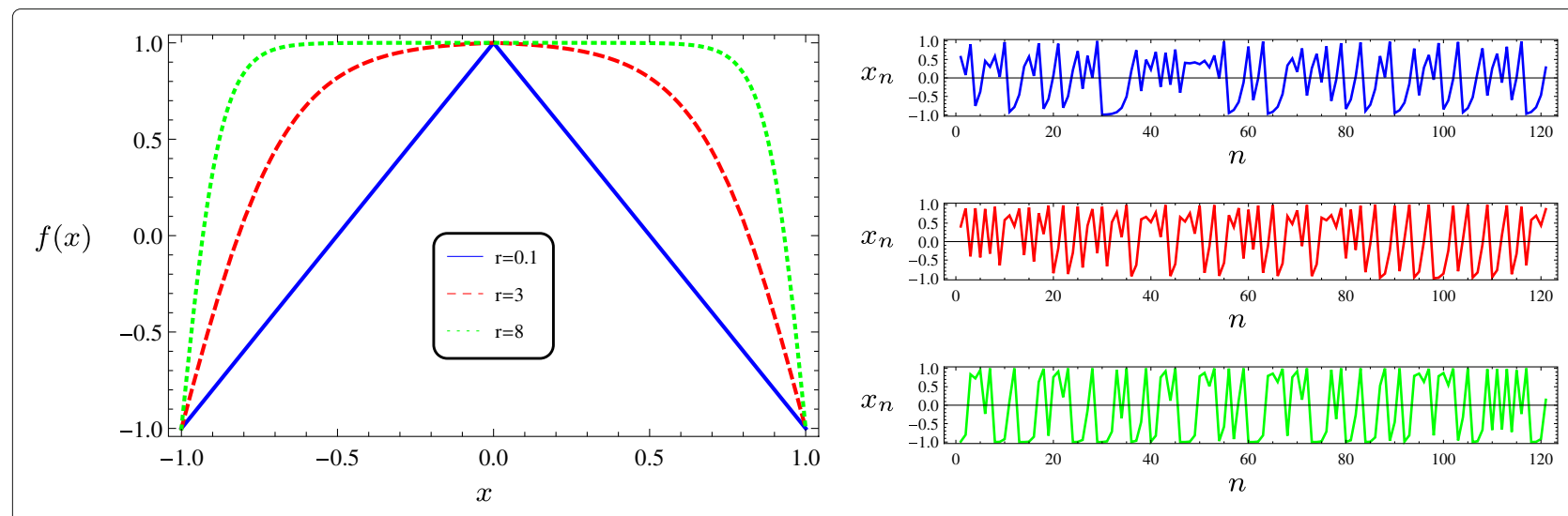

Fig. 1 The e-tanh map and its orbits for three values of $r, r=0.1,3,8$

from a known final condition $x_{N}$ (obtained through (6) with fixed parameters $b$ and $r$ ), that is

$$
x_{k}=f_{s_{k}}^{-1}\left(x_{k+1}\right), \quad k=N-1, \cdots, 0 .
$$

In this chaotic modulation scheme, if $s_{k}=0$, the first equation of (6) is used in the iterative process. Otherwise, if $s_{k}=1$, the second equation is used. The received signal at the $k$ th interval is given by

$$
r_{k}=x_{k}+n_{k}
$$

where $n_{k}$ in (9) is a zero-mean Gaussian random variable with variance $N_{0} / 2$. The decoder implements the Viterbi algorithm and is called in [24] by Viterbi decoding algorithm for chaotic signals (VDAC). The decoder uses a simplified trellis with two states and two outgoing branches from each state corresponding to each information symbol. The trellis structure at the $k$ th interval is shown in Fig. 6 . At the $k$ th interval, the $i$ th state stores the chaotic sample obtained by iterating the map backwards starting from $x_{N}$ and following the surviving path until this state, denoted by $\hat{x}_{k}^{i}$, and the corresponding metric associated to this state, $C_{k}^{i}$. The branch metric associated to the $j$ th branch starting from state $i$ is

$$
c_{i j}[k]=\left|r_{k}-f_{j}^{-1}\left(\hat{x}_{k}^{i}\right)\right|^{2} .
$$

Then, the decoding algorithm finds the metric at the $(k-1)$-th interval $\left(C_{k-1}^{i}\right)$ and the corresponding survivor paths and processes the received sequence in a recursive manner to find the path through the trellis with the smallest metric.

Let $\bar{E}_{x}$ be the average energy of the transmitted sequence. The signal-to-noise ratio (SNR) is defined as

$$
\mathrm{SNR}=\frac{\bar{E}_{x}}{N_{0}} .
$$

Figure 7 shows the bit error rate (BER) versus SNR for the e-tanh map for $r=8,10,12$. The BPSK curve is also shown for reference purposes. When $r$ increases, the performance is very close to that of the BPSK, indicating that this map achieves its goal of providing a guard region (in a statistical sense) specified by the parameter $r$ without suppressing orbits. The performance of the o-tanh is very similar (curves not shown). The variation of the parameter
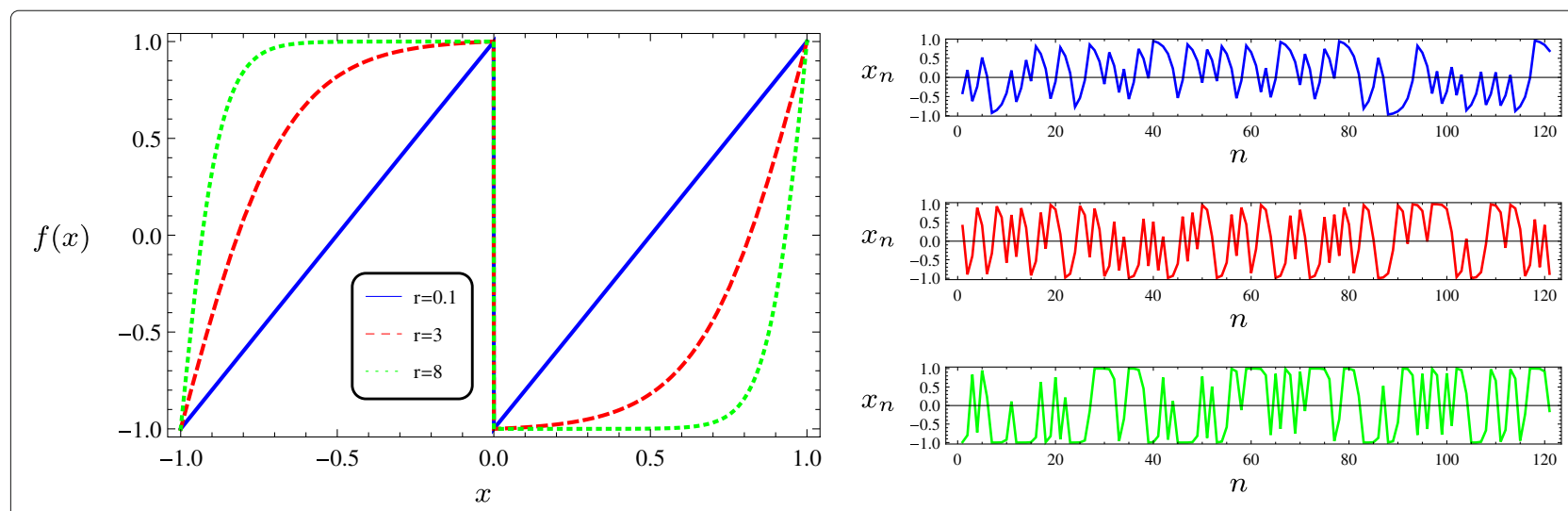

Fig. 2 The o-tanh map and its orbits for three values of $r, r=0.1,3,8$ 

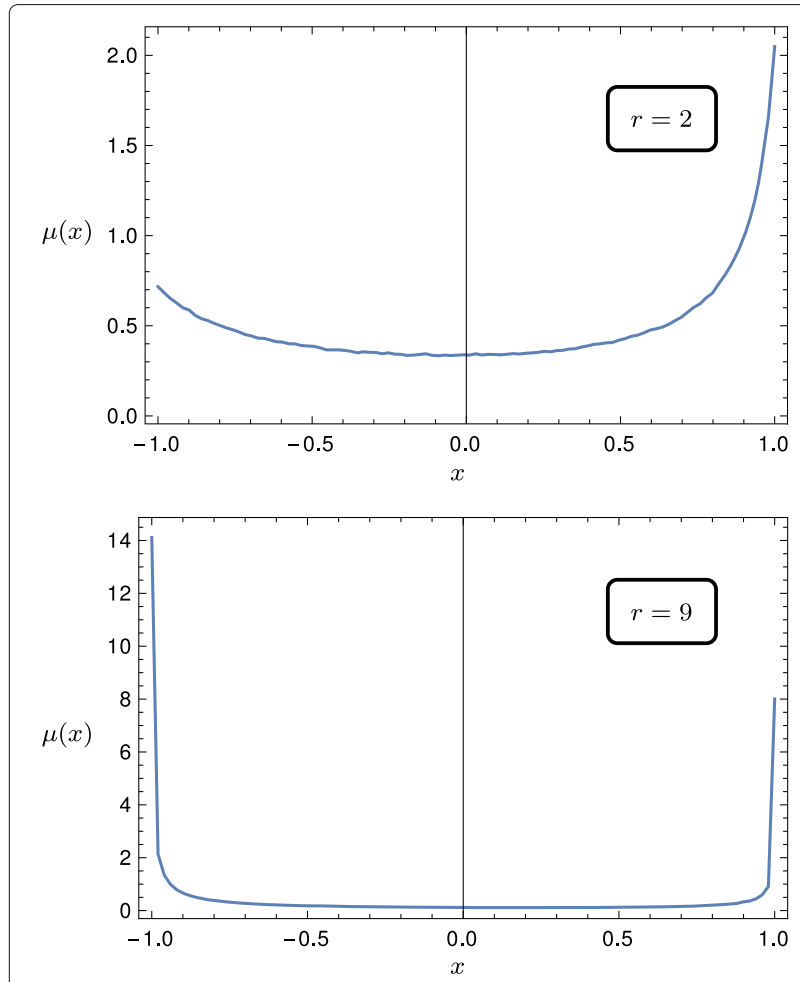

Fig. 3 Invariant distribution for the e-tanh map for $r=2$ and $r=9$
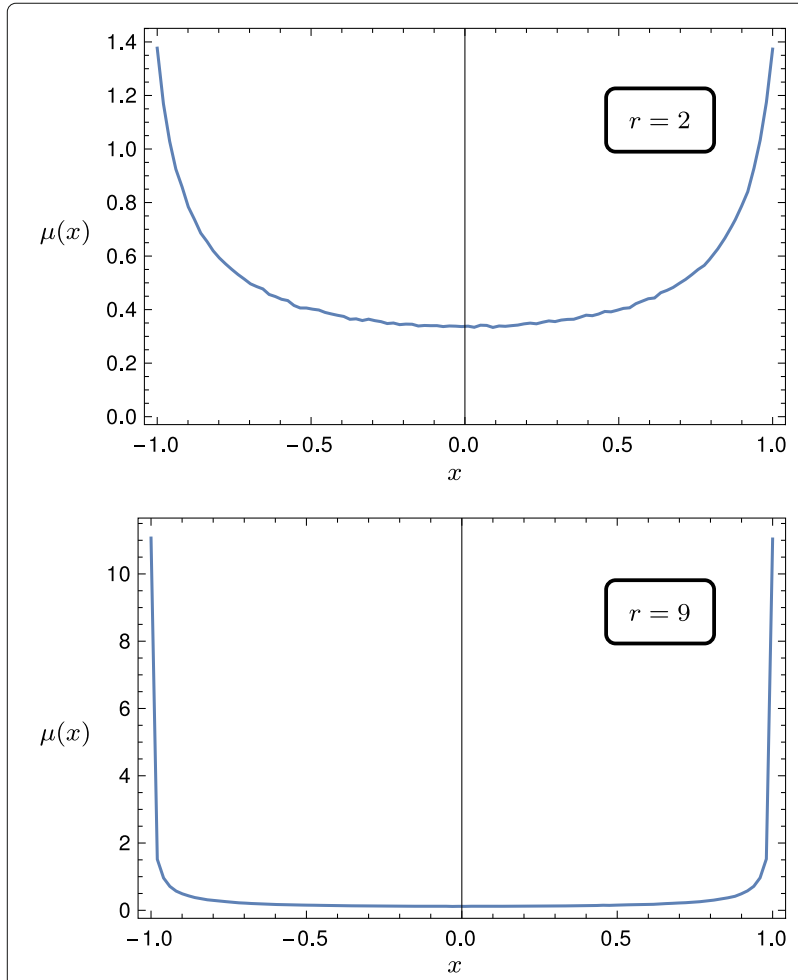

Fig. 4 Invariant distribution for the o-tanh map for $r=2$ and $r=9$

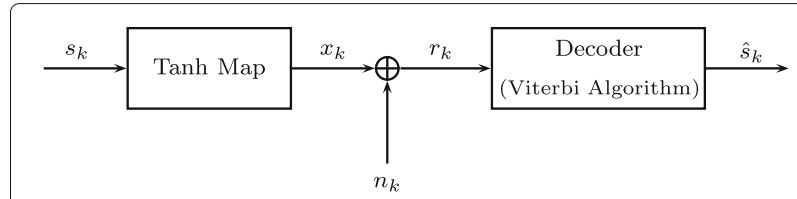

Fig. 5 Block diagram of a chaotic communication system based on the tanh map

$r$ has an important impact on the BER performance, and we will show in the next section that this variation has no significant impact on the Lyapunov exponent of this map.

In the next sections, we study the chaotic behavior of the proposed tanh map given in (4).

\section{Lyapunov exponent and orbit diagram for the tanh map}

The Lyapunov exponent quantifies the degree of separation of neighboring orbits. A chaotic system has a positive Lyapunov exponent, so infinitesimally close orbits on the attractor separate exponentially fast, on average. For an orbit $\left\{x_{i}\right\}_{i=0}^{\infty}$, the Lyapunov exponent of a one-dimensional discrete map is defined as $[1,9]$

$$
\lambda=\lim _{N \rightarrow \infty} \frac{1}{N} \sum_{i=0}^{N-1} \ln \left|f^{\prime}\left(x_{i}\right)\right|
$$

where $f^{\prime}(x)$ is the derivative of $f(x)$. The Lyapunov exponent can be calculated exactly when the map is ergodic and its invariant measure $\mu(x)$ is known. In this case, the ergodicity property implies that temporal averages equals spatial averages on the phase space with respect to the invariant measure and the Lyapunov exponent is expressed as [30]

$$
\lambda=\int \ln \left(\left|f^{\prime}(x)\right|\right) \mu(x) d x
$$

We show in the following that the Lyapunov exponent of the tanh map does not depend on $b$.

Consider the definition given in (10). Let $\left\{x_{i}\right\}_{i=0}^{\infty}$ and $\left\{y_{i}\right\}_{i=0}^{\infty}$ be two orbits generated by the e-tanh map and o$\tanh$ map, respectively. Suppose that $x_{0}=y_{0}$ and $\left|x_{\ell}\right| \neq$

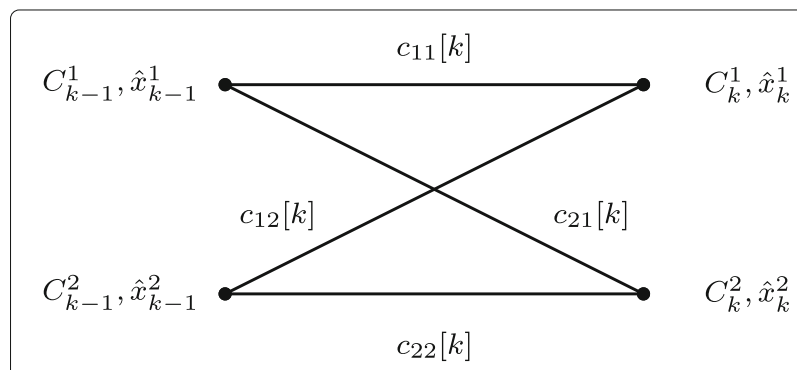

Fig. $6 \mathrm{~A}$ trellis section used by the Viterbi algorithm to decode the chaotic sequence 


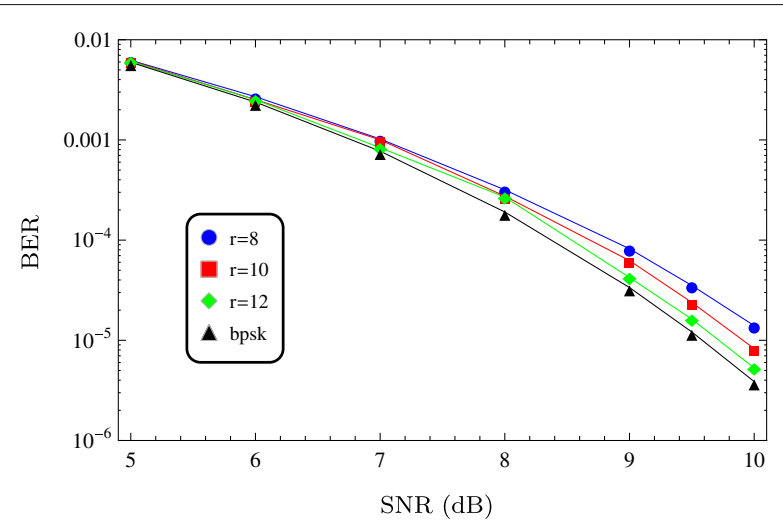

Fig. 7 BER versus SNR for the backward iteration of the e-tanh chaotic map with $r=8,10,12$

$\left|y_{\ell}\right|$ for a positive integer $\ell$. Thus, there exists a minimum integer $i, 0<i<\ell$, such that $\left|x_{i+1}\right| \neq\left|y_{i+1}\right|$. We can see from (4) that $\left|x_{i+1}\right|$ and $\left|y_{i+1}\right|$ are distinct only if $x_{i} \neq y_{i}$, but since $\left|x_{i}\right|=\left|y_{i}\right|$, thus $y_{i}=-x_{i}$. Without loss of generality, suppose $x_{i} \geq 0$, then $\left|x_{i+1}\right|=\left|f\left(x_{i}\right)\right|=$ $\left|e \cdot \tanh \left(-r\left(x_{i}-1\right)\right)-1\right|=\left|e \cdot \tanh \left(r\left(-x_{i}+1\right)\right)-1\right|=\mid e \cdot$ $\tanh \left(r\left(y_{i}+1\right)\right)-1|=| f\left(y_{i}\right)|=| y_{i+1} \mid$. Consequently, starting from the same initial conditions $\left(x_{0}=y_{0}\right)$, we conclude that $\left\{\left|x_{i}\right|\right\}_{i=0}^{\ell}=\left\{\left|y_{i}\right|\right\}_{i=0}^{\ell}$ for all $\ell>0$. Next, we proof that the absolute value of the derivative of the tanh map depends on only of the absolute value of the argument. Suppose again that $x_{i} \geq 0$ and that $f(x)$ is the e-tanh map, thus $\left|f^{\prime}\left(x_{i}\right)\right|=\left|e \cdot(-r) \cdot \operatorname{sech}^{2}\left(-r\left(x_{i}-1\right)\right)\right|=$ $\left|e \cdot r \cdot \operatorname{sech}^{2}\left(r\left(-x_{i}+1\right)\right)\right|=\left|f^{\prime}\left(-x_{i}\right)\right|$, which is also true for the o-tanh map. Therefore, $\left\{\left|x_{i}\right|\right\}_{i=0}^{\ell}=\left\{\left|y_{i}\right|\right\}_{i=0}^{\ell}$ implies that $\left\{\left|f^{\prime}\left(x_{i}\right)\right|\right\}_{i=0}^{\ell}=\left\{\left|f^{\prime}\left(y_{i}\right)\right|\right\}_{i=0}^{\ell}$.

We conclude from (10) that the Lyapunov exponent of the tanh map is invariant with the parameter $b$.

We compute the Lyapunov exponent of the tanh map using (10) from a finite orbit with $N=10,000$ points, discarding the first 200 points to eliminate the transient behavior. Figure 8 shows that the Lyapunov exponent of

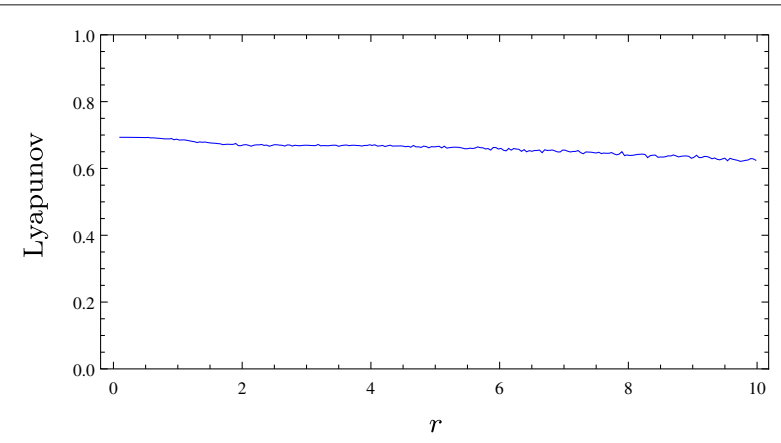

Fig. 8 Lyapunov exponent versus the parameter $r$ for the tanh map the tanh map is positive for the range of values of $r$ considered in this figure. It should be remarked that, if $r$ tends to zero, the value of the Lyapunov exponent is equal to that of the tent map (0.693), and it slightly decreases with the increase of $r$.

Otherwise, the Lyapunov exponent of the modified Bernoulli map defined in (2) is strongly dependent on its parameter $p$ and is expressed as [31]

$$
\lambda=(1-p) \ln \left(\frac{2}{1-p}\right)+p \ln \left(\frac{1}{p}\right) .
$$

For example, $\lambda=0.639$ for $p=0.8$ and $\lambda=0.394$ for $p=0.9$. The Lyapunov exponent of this map with $p=0.8$ is approximately the same of that of the tanh map with $r=12$. We fix $p$ e $r$ in each map and compare their BER in Fig. 9 using the same communication scenario discussed in the previous section. We also show in this figure that the case $p=0.9$ provides better BER performance than the case $p=0.8$ at the expense of a lower Lyapunov exponent. The curves in this figure show that the tanh map with $r=$ 12 outperforms the modified Bernoulli map for these two values of $p$.

The orbit diagram of the e-tanh map is shown in Fig. 10, as complementary information, and also illustrates the chaotic behavior of this map. It should be noted that the points concentrate around extremal values \pm 1 when $r>$ 2.0. A similar behavior is observed for the o-tanh map (figure not shown) with a symmetric distribution of points around $x=0$, as is indicated in Fig. 4.

\section{Structural measures}

A dynamical system may be analyzed from a variety of perspectives, as is intended by the definitions of structural measures, e.g., phase space dimension, topological dimension, fractal dimension, information dimension, information entropy, and embedding dimension [32]. The choice of an appropriate performance measure depends

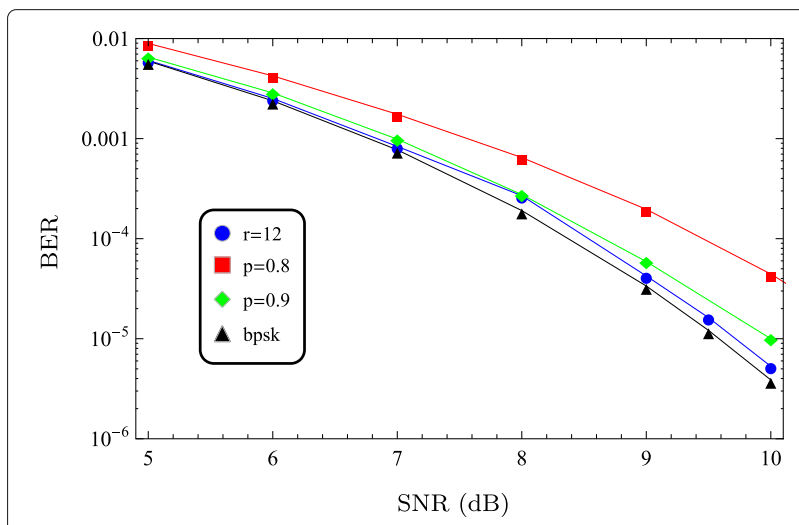

Fig. 9 BER versus SNR for the backward iteration of the modified Bernoulli map with $p=0.8, p=0.9$ and the e-tanh chaotic map with $r=12$ 


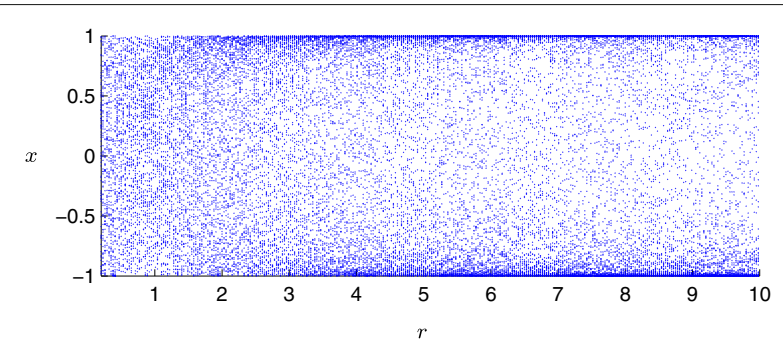

Fig. 10 Orbit diagram versus the parameter $r$ for the e-tanh map

on the intended application. In this section, we first review three well-known generalized measures that capture the local structure of the attractor [33, 34]. Next, we define a new measure, called spread rate, motivated by the performance gain observed in the chaotic communication system considered in Section 4. This gain is achieved by exploring the concentration of points observed in the invariant distribution of the tanh map. So, the spread rate measures the concentration of points on the attractor as a consequence of the dynamical process of a chaotic map.

\subsection{Measures on the attractor}

Let $\left\{x_{i}\right\}_{i=0}^{N}$ be an orbit of a chaotic map and consider the coverage of its attractor by hypercubes of edge length $\ell$. The number of hypercubes that contain a piece of the attractor is $M(\ell)$. A set of measures that address the local structure of fractal attractors are based on these concepts. As a first example, we consider de fractal (HausdorffBesikovich) dimension $D$ [35], a purely geometric measure, that characterizes the growth of the minimal number of hypercubes of edge length $\ell$ needed for covering the attractor as $\ell \rightarrow 0$. It can be shown that $M(\ell) \simeq \ell^{-D}$. This measure is independent of the frequency with which a typical trajectory visits the various parts of the attractor. Other aspect to consider is the computational hardness for calculating $D$.

An alternative for $D$ that captures the pattern of occupation of the attractor is the information entropy, $S(\ell)$. It may be understood as the information gained by an observer by measuring the actual state $x_{i}$ of the system with accuracy $\ell$, assuming that he knows all properties of the system except the initial state $x_{0}$. The information entropy can be written as $[32,36]$

$$
S(\ell)=-\sum_{i=1}^{M(\ell)} p_{i} \ln p_{i} \simeq S_{0}-\sigma \ln \ell
$$

where $p_{i}=\lim _{N \rightarrow \infty} \frac{\mu_{i}}{N}$, for a run length $N$ with $\mu_{i}$ occurrences in the $i$ th hypercube, and $\sigma$ is called the information dimension. It is worth noting that $S_{0}$ is a constant related to the differential entropy and is obtained as the limit (as $\ell \rightarrow 0$ ) of the relative entropy between the distribution of occurrences of hypercubes over the attractor and that of the uniform occupation of the hypercubes $[37,38]$. Moreover, in the context of lossless analog compression, the information dimension is interpreted as the entropy rate of a dyadic expansion of the system [38], a relation that brings a intrinsic connection between information systems and dynamical systems. For a uniform distribution, $p_{i}=1 / M(\ell)$, the entropy is maximum and the information dimension is equal to the fractal dimension [33, 34]. The computational complexity for the calculation of the information dimension is similar to that of the fractal dimension [32].

Due to the exponential divergence of trajectories, most pairs $\left(x_{i}, x_{j}\right)$ with $i \neq j$ are dynamically uncorrelated pairs. However, the points lie on the attractor; thus, they are spatially correlated. This spacial correlation can be measured by the correlation integral $C(\ell)$ [39], defined according to $C(\ell)=\lim _{N \rightarrow \infty} \frac{1}{N^{2}} \times\left\{\right.$ number of pairs $(i, j)$ such that $\left.\left|x_{i}-x_{j}\right|<\ell\right\}$.

It is known that, for small hypercubes of edge length $\ell$, the growth $C(\ell) \sim \ell^{v}$ is observed [39], where $v$ is a constant that can be taken as a measure of the local structure of the strange attractor.

We revise in this subsection three classes of measures, defined on the attractor, that capture three distinct structural aspects of the attractor: geometric, spacial distribution, spacial correlation. We propose in the next subsection a new measure that captures the concentration of points on the attractor of a one-dimensional chaotic system defined by $f(x)$, as is the case of Bernoulli map, tanh map, tent map, logistic map, and others. It is worth mentioning that the proposed measure only captures the dynamics aspects that generate concentration of points, what is obtained by the suppression of the effects of geometry (static aspects). Moreover, it is defined on the graph of $f(x)$ and not on the attractor of the map.

\subsection{Spread rate}

The spread rate $(\mathcal{S R})$ is a structural measure that captures the concentration of points induced by the dynamics of a one-dimension map $f(x)$. Specifically, it quantifies the concentration of points on the attractor with the compensation of the stretching and squeezing generated by the change of the derivative of $f(x)$ along the curve, which is the geometric mechanism responsible for the concentration of points on the attractor. To suppress the geometric influence on the compression effect, the evolution of the system is analyzed on the graph of $f(x)$, and not on 
its domain, where the attractor is embedded. This permits to compare the concentration induced by different dynamics. For parameterized maps, it permits to identify if the concentration property of the dynamics effectively changes with the variation of the control parameter.

The spread rate is defined as the percentage of effectively visited regions of the curve $f(x)$. A region is called effectively visited if its length is shorter than a threshold $s_{\text {th }}$, that is the arc length between two consecutive points over the curve for a uniform distribution of points. Therefore an orbit of length $N$ of a map $f(x)$ has threshold $s_{\text {th }} \triangleq L / N$, where $L$ is the length of the curve and is obtained from the well-known formula from calculus

$$
L=\int_{-1}^{1} \sqrt{1+\left[f^{\prime}(x)\right]^{2}} d x
$$

The ratio of the total length of effectively visited regions to the length of $f(x)$ specifies the $\mathcal{S R}$. For an arbitrary orbit $\left\{x_{i}\right\}_{i=0}^{N-1}$, not necessarily homogeneously distributed, let $\left\{\tilde{x}_{i}\right\}_{i=0}^{N-1}$ be its ordered version, such that $\tilde{x}_{i}<\tilde{x}_{i+1}$. The partition induced on the curve of $f(x)$ by $\left\{\tilde{x}_{i}\right\}_{i=0}^{N-1}$ is given by the sequence of arcs $\left\{s_{i}\right\}_{i=1}^{N-1}$, such that the $k$ th arc is delimited by the pairs $\left(\tilde{x}_{i}, f\left(\tilde{x}_{i}\right)\right)$ and $\left(\tilde{x}_{i+1}, f\left(\tilde{x}_{i+1}\right)\right)$ and has length

$$
s_{k}=\int_{\tilde{x}_{k-1}}^{\tilde{x}_{k}} \sqrt{1+\left[f^{\prime}(x)\right]^{2}} d x .
$$

The length $s_{k}$ is counted as part of the effective occupied length $s$ if $s_{k} \leq s_{\text {th }}$, thus

$$
s=\sum_{\substack{s_{k} \leq s_{\mathrm{th}} \\ 1 \leq k \leq N-1}} s_{k} .
$$

Finally, we define $\mathcal{S} \mathcal{R}$ as

$$
\mathcal{S R} \triangleq \frac{s}{L}=\frac{1}{N s_{\mathrm{th}}} \sum_{\substack{s_{k} \leq s_{\mathrm{th}} \\ 1 \leq k \leq N-1}} s_{k}=\frac{1}{N} \sum_{\substack{s_{k} \leq s_{\mathrm{th}} \\ 1 \leq k \leq N-1}} \frac{s_{k}}{s_{\mathrm{th}}} .
$$

We observe by simulations that, for the same initial conditions, the tanh map generates distinct sets of arc lengths for $b=0$ and $b=1$, but there is a statistical convergence of the $\mathcal{S} \mathcal{R}$ curves for these two values of $b$. Thus, the ergodic properties of the tanh map measured by $\mathcal{S} \mathcal{R}$ are invariant on $b$.

The $\mathcal{S R}$ versus $r$ for the tanh map is shown in Fig. 11. For values of $r$ from 0 to around 2, the $\mathcal{S R}$ curve is approximately flat and shows that about $26 \%$ of the length of $f(x)$ amounts for the overall effectively visited regions, thus about a quarter of the attractor is effectively occupied. The flatness of the $\mathcal{S R}$ curve for this range of $r$ indicates that the concentration (around \pm 1 ) shown in the upper right

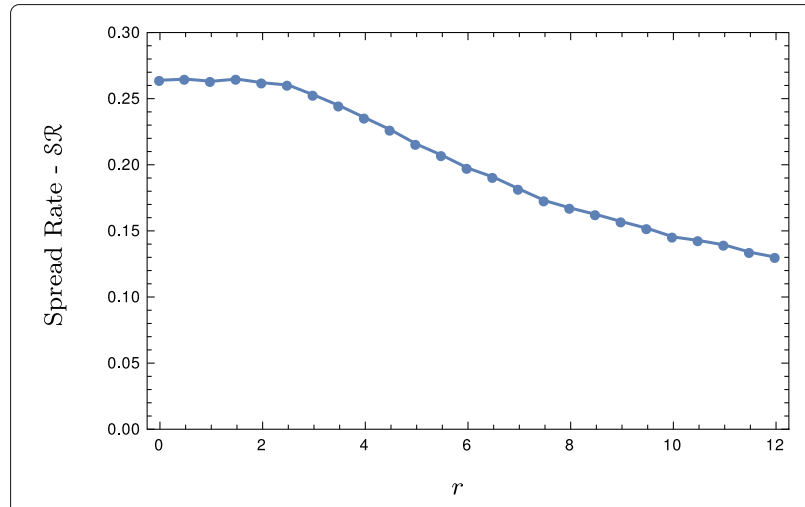

Fig. $11 \mathcal{S R}$ for the tanh map as a function of the parameter $r$

plot of Figs. 3 and 4 for $r=2$ is due to the geometry of the curve and not to the dynamic. For $r>2$, SR presents an approximately linear decrease with angular coefficient around -0.016 or $6.2 \%$ of the initial value of $\mathcal{S R}$. Thus, $\mathcal{S} \mathcal{R}$ reduces by $6.2 \%$ per unit of $r$ from $r=2$ until $r=10$. The negative slope of the $\mathcal{S R}$ for this range of $r$ corresponds to an increase of the compression induced by the dynamics, and so, beyond the compression associated to the geometry. We also compute the percentage of points within the effectively visited region. It changes from $63 \%$ for $r=2$ to $72 \%$ for $r=10$. Thus, the increase of $r$ results in a growing number of points into a smaller region of the attractor.

The $\mathcal{S} \mathcal{R}$ versus the parameter $p$ for the modified Bernoulli map defined in (2) is given in Fig. 12. We observe the flatness of the curve for $0.2<p<0.8$, with value approximately equal to the maximum value of the $\mathcal{S} \mathcal{R}$ for the tanh map, that occurs for $0<r<2$. Therefore, in this flat region, the variation of $p$ has no impact on the compression of the map due to the dynamics. Otherwise, for $p \leq 0.2$ or $p \geq 0.8$, the compression due to the dynamics decreases.

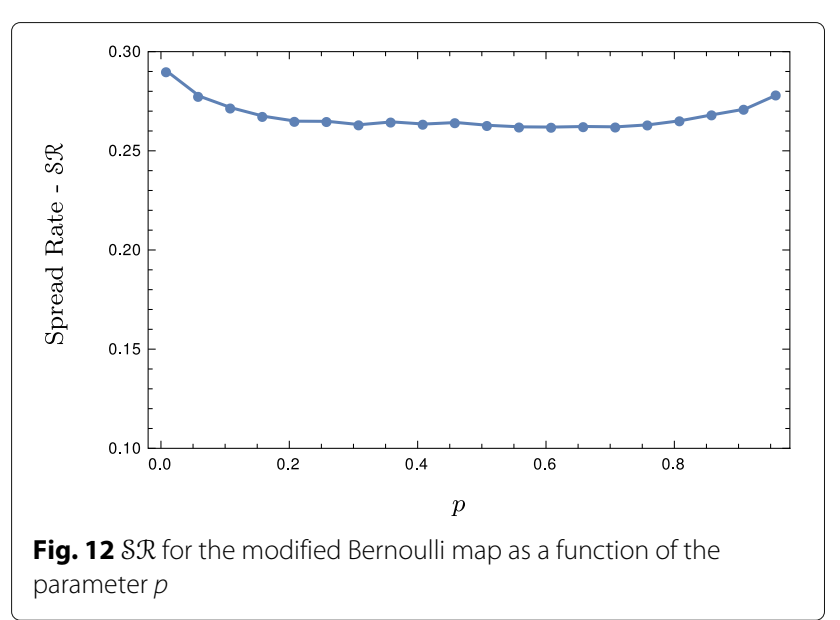




\section{Conclusions}

The investigation of the chaotic properties of the tanh map reveals that it is a generalization of the well studied tent and Bernoulli maps. The concentration of points of an orbit around +1 and -1 can be controlled by $r$, and for high enough $r$, the system rarely assumes values around zero. The BER analysis allows us to design symbolic dynamics-based chaotic modulators with a guard region without degenerating the chaos by suppressing orbits. Moreover, the variation of $r$ has slight impact on Lyapunov exponent of the tanh map. Thus, this map is a promising candidate for chaos communication. We also propose a new measure, called spread rate, to evaluate the level of concentration of the dynamic on regions over the attractor. We compare the performance of the tanh map with that of the modified modified Bernoulli map. An interesting future direction is to extend this comparison to modified versions of the tent map, such as the maps defined in [40-42]. Another direction for future research is the calculation of the invariant measure of the tanh map. The tanh map can be implemented by using an emittercoupled pair. Thus, a chaotic system with the tanh map is a good candidate for low weight applications such as sensor networks and in-building wireless devices for UWB communication.

\section{Funding}

This work was supported in part by FACEPE (under Grants APQ-0291-3.04/14 and APQ-0683-3.04/12) and CNPq (under Grant 471729/2012-4).

Received: 25 December 2015 Accepted: 27 September 2016

Published online: 17 November 2016

\section{References}

1. K Alligood, T Sauer, J Yorke, Chaos: An Introduction to Dynamical Systems. (Springer, NY, 1997)

2. L Kocarev, S Lian, Chaos-based Cryptography: Theory, Algorithms and Applications, Studies in Computational Intelligence. (Springer, 2011)

3. A Beirami, $\mathrm{H}$ Nejati, A framework for investigating the performance of chaotic-map truly random number generators. IEEE Trans.Circ. Syst. II: Express Briefs. 60, 446-450 (2013)

4. S Callegari, R Rovatti, G Setti, Embeddable ADC-based true random number generator for cryptographic applications exploiting nonlinear signal processing and chaos. IEEE Trans.Sig. Process. 53, 793-805 (2005)

5. F Liu, CK Wu, Robust visual cryptography-based watermarking scheme for multiple cover images and multiple owners. IET Inf.Secur. 5, 121-128 (2011)

6. B Jovic, Chaos-based BPSK communication system. Electron.Lett. 51, 630-632 (2015)

7. W Xu, L Wang, G Chen, Performance analysis of the CS-DCSK/BPSK communication system. IEEE Trans.Circuits Syst. I: Regular Papers. 61, 2624-2633 (2014)

8. M Hasler, G Mazzini, M Ogorzalek, R Rovatti, G Setti, Scanning the special issue - special issue on applications of nonlinear dynamics to electronic and information engineering. Proc.IEEE. 90, 631-640 (2002)

9. S Strogatz, Nonlinear Dynamics and Chaos with Applications to Physics, Biology, Chemistry, and Engineering, Studies in Nonlinearity Series. (Westview Press, 2001)

10. G Kaddoum, F Shokraneh, Analog network coding for multi-user multi-carrier differential chaos shift keying communication system. IEEE Trans.Wirel. Comm. 14, 1492-1505 (2015)

11. F Lau, C Tse, Chaos-Based Digital Communication Systems, Engineering online library. (Springer, 2010)
12. G Kaddoum, J Olivain, G Beaufort Samson, P Giard, F Gagnon, in International Symposium on Wireless Communication Systems (ISWCS). Implementation of a differential chaos shift keying communication system in gnu radio, (2012), pp. 934-938

13. P Stavroulakis, Chaos Applications in Telecommunications. (Taylor \& Francis, 2005)

14. AP Kurian, S Puthusserypady, SM Htut, Performance enhancement of DS/CDMA system using chaotic complex spreading sequence. IEEE Trans.Wirel. Commun. 4, 984-989 (2005)

15. Z Galias, GM Maggio, Quadrature chaos-shift keying: theory and performance analysis. IEEE Trans.Circ. Syst. I: Fundam. Theory Appl. 48, 1510-1519 (2001)

16. H Yang, GP Jiang, High-efficiency differential-chaos-shift-keying scheme for chaos-based noncoherent communication. IEEE Trans.Circ. Syst. II: Express Briefs. 59, 312-316 (2012)

17. G Kaddoum, FD Richardson, F Gagnon, Design and analysis of a multi-carrier differential chaos shift keying communication system. IEEE Trans.Commun. 61, 3281-3291 (2013)

18. G Kaddoum, Design and performance analysis of a multiuser OFDM based differential chaos shift keying communication system. IEEE Trans.Commun. 64, 249-260 (2016)

19. G Kaddoum, E Soujeri, C Arcila, K Eshteiwi, I-DCSK: An improved noncoherent communication system architecture. IEEE Trans.Circ. Syst. II: Express Briefs. 62, 901-905 (2015)

20. G Kaddoum, E Soujeri, Y Nijsure, Design of a short reference noncoherent chaos-based communication systems. IEEE Trans.Commun. 64, 680-689 (2016)

21. J Schweizer, T Schimming, Symbolic dynamics for processing chaotic signals.I. Noise reduction of chaotic sequences. IEEE Trans. Circuits Syst. I, Fundam. Theory Appl. 48, 1269-1282 (2001)

22. S Hayes, C Grebogi, E Ott, Communicating with chaos. Phys.Rev. Lett. 70, 3031-3034 (1993)

23. C Williams, Robust chaotic communications exploiting waveform diversity.Part 1: Correlation detection and implicit coding. IET Commun. 2 1213-1222 (2008)

24. D Luengo, I Santamaria, in IEEE Vehicular Technology, Conference VTC 2005-Spring). Secure communications using OFDM with chaotic modulation in the subcarriers, vol. 2, (2005), pp. 1022-1026

25. G Kaddoum, M Vu, F Gagnon, in IEEE International Symposium on, Circuits and Systems (ISCAS). Chaotic symbolic dynamics modulation in MIMO systems, (2012), pp. 157-160

26. G Kaddoum, G Gagnon, F Gagnon, Spread spectrum communication system with sequence synchronization unit using chaotic symbolic dynamics modulation. Int.J. Bifurcat. Chaos. 23, 1350019.1-1350019.14 (2013)

27. G Kaddoum, F Gagnon, D Couillard, in International Conference on, Signal Processing and Communication Systems (ICSPCS). An enhanced spectral efficiency chaos-based symbolic dynamics transceiver design, (2012), pp. 1-6

28. B Hao, W Zheng, Applied Symbolic Dynamics and Chaos. Directions in chaos. (World Scientific, 1998)

29. D Pederson, K Mayaram, Analog Integrated Circuits for Communication: Principles, Simulation, and Design. (Kluwer Academic Publishers, 1991)

30. C Robinson, Dynamical Systems Stability, Symbolic Dynamics and Chaos. (CRC Press, 1995)

31. K Feltekh, D Fournier-Prunaret, S Belghith, Analytical expressions for power spectral density issued from one-dimensional continuous piecewise linear maps with three slopes. Signal Process. 94, 149-157 (2014)

32. BB Ali, H Nayfeh, Applied Nonlinear Dynamics. Wiley Series in Nonlinear Science. (Wiley-VCH, 1995)

33. H Hentschel, I Procaccia, The infinite number of generalized dimensions of fractals and strange attractors. Physica D: Nonlinear Phenom. 8(3), 435-444 (1983)

34. P Grassberger, Generalized dimensions of strange attractors. Phys.Lett. A. 97(6), 227-230 (1983)

35. J Guckenheimer, Noise in chaotic systems. Nature. 298, 358-361 (1982)

36. A Rényi, On the dimension and entropy of probability distributions. Acta Math. Acad. Sci. Hung. 10(1), 193-215 (1959)

37. T Cover, J Thomas, Elements of Information Theory. (Wiley, 2012)

38. Y Wu, S Verdu, Rényi information dimension: fundamental limits of almost lossless analog compression. IEEE Trans.Inf. Theory. 56, 3721-3748 (2010) 
39. P Grassberger, I Procaccia, Characterization of strange attractors. Phys.Rev. Lett. 50, 346-349 (1983)

40. S Callegari, G Setti, PJ Langlois, in Proc. 1997 IEEE International Symposium on, Circuits and Systems. A CMOS tailed tent map for the generation of uniformly distributed chaotic sequences, vol. 2, (1997), pp. 781-784

41. H Nejati, A Beirami, Y Massoud, in 51st Midwest, Symposium on Circuits and Systems. A realizable modified tent map for true random number generation, (2008), pp. 621-624

42. H Nejati, A Beirami, WH Ali, Discrete-time chaotic-map truly random number generators: design, implementation, and variability analysis of the zigzag map. Analog Integr.Circ. Sig. Process. 73(1), 363-374 (2012)

\section{Submit your manuscript to a SpringerOpen ${ }^{\circ}$} journal and benefit from:

- Convenient online submission

- Rigorous peer review

- Immediate publication on acceptance

- Open access: articles freely available online

- High visibility within the field

- Retaining the copyright to your article

Submit your next manuscript at $\gg$ springeropen.com 